\title{
Gauged six-dimensional supergravity from warped IIB reductions
}

\section{Junho Hong, James T. Liu and Daniel R. Mayerson}

Leinweber Center for Theoretical Physics, Randall Laboratory of Physics,

The University of Michigan,

Ann Arbor, MI 48109-1040, U.S.A.

E-mail: junhoh@umich.edu, jimliu@umich.edu, drmayer@umich.edu

ABSTRACT: We find a family of complete non-linear Kaluza-Klein reduction ansätze from type IIB supergravity to Romans' $6 \mathrm{D} F(4)$ gauged supergravity in the bosonic sector. The reduction is over a sphere $S^{2}$ and a Riemann surface $\Sigma$, and depends on a pair of arbitrary locally holomorphic functions $\mathcal{A}_{ \pm}$on $\Sigma$. This family of reductions is inspired by the recent construction of $1 / 2$ BPS supersymmetric warped $A d S_{6}$ solutions of IIB supergravity that depend on these same functions $\mathcal{A}_{ \pm}$.

KEYWORDS: Supergravity Models, Superstring Vacua, D-branes

ArXiv ePrint: 1808.04301 


\section{Contents}

$\begin{array}{llr}1 & \text { Introduction } & 1\end{array}$

2 IIB supergravity and warped $A d S_{6} \times S^{2} \times \Sigma$ vacua 3

2.1 Type IIB supergravity 3

2.2 A family of warped $A d S_{6}$ solutions 4

$3 \quad 6 D$ supergravity and the reduction ansatz $\quad 5$

$\begin{array}{lll}3.1 & F(4) \text { gauged supergravity } & 6\end{array}$

$\begin{array}{lll}3.2 & \text { Generalized reduction ansatz } & 7\end{array}$

4 Discussion $\quad 8$

$\begin{array}{ll}4.1 \text { Holography } & 9\end{array}$

$\begin{array}{ll}\text { A Conventions } & 10\end{array}$

B Mapping between different 10D IIB conventions 11

C Checking the 10D EOMs 12

\section{Introduction}

Supergravity solutions of the form $A d S_{d} \times S^{n}$ have long been known, and have particular importance in the context of AdS/CFT. Perhaps the most familiar cases are those associated with the near-horizon geometry of M2, D3 and M5 branes. In contrast, the case of $A d S_{6} / C F T_{5}$ has been less well explored, in part because it does not admit a single brane interpretation. Nevertheless, there has been a recent resurgence of interest in this case, driven both on the field theory and the gravity sides of the duality. In contrast with the more familiar cases, holography in this dimension is interesting as there are no maximally supersymmetric 5D SCFTs (with 16 Poincaré supercharges and 16 superconformal supercharges). In particular, the maximal possible amount of supersymmetry in $5 \mathrm{D}$ is $8+8$ supercharges [1]. The superconformal algebra in $5 \mathrm{D}$ is then based on the Lie superalgebra $F(4)$ with maximal bosonic subalgebra $\mathrm{SO}(2,5) \times \mathrm{SO}(3)$ [2]. The natural six-dimensional dual is then $F(4)$ gauged supergravity which is a non-chiral theory with 16 real supercharges [3].

Of course, a string theory realization of $A d S_{6} / C F T_{5}$ starts not in six dimensions, but rather with 10 or 11 dimensional supergravity (in its low-energy limit) compactified to $A d S_{6}$ times some internal manifold. Such backgrounds can preserve at most half of the total supersymmetries [4], in accordance with the $C F T_{5}$ picture. Until recently, string theory realizations of such $A d S_{6}$ duals have been hard to come by, with the prime example being a 
construction of stacks of D4-branes, D8-branes, and O8-planes in type IIA string theory [58]. The presence of D8-branes means that the supergravity background is obtained in the massive IIA theory.

In type IIB, $(p, q)$ five-brane webs $[9,10]$ with D7-branes added [11] can realize large classes of 5D SCFTs. The holographic dual of these five-brane webs corresponds to supersymmetric $A d S_{6}$ solutions of IIB supergravity. The Killing spinor equations for this system were investigated in $[12,13]$ and reduced to a pair of coupled PDEs, and a complete family of local solutions was constructed in [14-16]. The local $A d S_{6}$ solutions were found by studying the IIB Killing spinor equations, which led to a family of solutions of the form $A d S_{6} \times S^{2}$ warped over a Riemann surface $\Sigma$; remarkably, these solutions are completely determined by a pair of holomorphic functions $\mathcal{A}_{ \pm}$on $\Sigma$ which can be chosen freely up to global regularity conditions.

More generally, the $A d S_{6}$ solutions of [14] ought to be viewed as vacuum solutions of six-dimensional $F(4)$ gauged supergravity obtained by reducing ten-dimensional IIB supergravity on $S^{2} \times \Sigma$. Curiously, the existence of a whole family of solutions suggests that the lifting of $F(4)$ gauged supergravity to ten dimensions is far from unique. This is in contrast to the 32 supercharge cases such as gauged $\mathcal{N}=8$ supergravity in fivedimensions, which has a unique lift to IIB supergravity on $S^{5}$. Before this family of IIB vacua was discovered, a full non-linear Kaluza-Klein reduction of massive IIA supergravity to $F(4)$ gauged supergravity on (one hemisphere of) $S^{4}$ was obtained in [17]. This was subsequently dualized to a IIB reduction in [18] using non-abelian T-duality. In particular, the IIA reduction ansatz of [17] was based on $A d S_{6}$ times a squashed $S^{4}$ foliated by 3spheres, with the $\mathrm{SU}(2) R$-symmetry corresponding to the gauging of $\mathrm{SU}(2)_{L}$ inside the $\mathrm{SO}(4) \simeq \mathrm{SU}(2)_{L} \times \mathrm{SU}(2)_{R}$ isometry of $S^{3}$. Non-abelian T-dualizing then gives rise to a IIB reduction where the $S^{4}$ is replaced by $S^{2} \times \Sigma$, with the $R$-symmetry now corresponding to the $\mathrm{SO}(3)$ isometry of $S^{2}$. However, this procedure gives rise to only a single background, first obtained in [19], and not the entire family of solutions constructed in [14].

In this paper, we present a complete family of consistent truncations of IIB supergravity to $F(4)$ gauged supergravity parametrized by the same holomorphic functions $\mathcal{A}_{ \pm}$of [14]. This generalizes the family of $A d S_{6}$ vacua into complete reduction ansätze, in agreement with the conjecture that any supersymmetric vacuum solution can be extended to a consistent truncation incorporating the full lower-dimensional supergravity multiplet [20, 21]. (General half-maximal consistent truncations were recently considered in the framework of exceptional field theory in [22].) The non-linear reduction to $F(4)$ gauged supergravity was inspired by the construction of [18], but is in fact much more general. Naturally, it incorporates the reduction ansatz of [18] as a special case, and we give the explicit functions $\mathcal{A}_{ \pm}$necessary to recover the results of [18] in section 4 .

The rest of the paper is structured as follows. In section 2, we review the basics of 10D type IIB supergravity, and briefly discuss the $1 / 2$ BPS $A d S_{6} \times S^{2} \times \Sigma$ solutions of [14] that depend on the pair of holomorphic functions $\mathcal{A}_{ \pm}$on $\Sigma$. Then, in section 3 , we discuss Romans' 6D $F(4)$ gauged supergravity and give our full non-linear reduction ansatz, which also depends on the holomorphic functions $\mathcal{A}_{ \pm}$. Finally, in section 4 , we discuss how our ansatz reduces to previous results in the literature in special cases, and the 
connection to $A d S_{6} / C F T_{5}$ holography. The appendices discuss our conventions for indices and coordinates on the various manifolds we consider (appendix A), the relation between different conventions for IIB supergravity (appendix B), and more details of checking the equations of motion on our ansatz (appendix C).

Main result. Our main result is the full non-linear Kaluza-Klein reduction ansatz given in section 3.2 in (3.7)-(3.10). On this ansatz, the 10D type IIB supergravity equations of motion (2.3) are equivalent to the 6D $F(4)$ gauged supergravity equations of motion (3.3). The reduction ansatz depends on a pair of unrestricted holomorphic functions $\mathcal{A}_{ \pm}$on the Riemann surface $\Sigma$.

Note added. After this work appeared on the arXiv, the work [23] was released, which also constructs the consistent truncation to $F(4)$ gauged supergravity.

\section{IIB supergravity and warped $\operatorname{AdS}_{6} \times S^{2} \times \Sigma$ vacua}

Before presenting the non-linear Kaluza-Klein ansatz, we quickly review the equations of motion of IIB supergravity and the family of supersymmetric $A d S_{6}$ solutions of [14]. This sets up our conventions and lays the groundwork for the reduction.

\subsection{Type IIB supergravity}

Type IIB supergravity is a chiral theory with 32 real supercharges. Because of the self-dual five-form, it does not admit a covariant Lagrangian formulation (although one can come close). Of course, for presenting the consistent truncation, we only need the equations of motion, which we give in the $\mathrm{SU}(1,1)$ formulation $[24,25]$.

The bosonic fields consist of the Einstein frame metric $g_{M N}$, a complex scalar with kinetic term $P_{1}$ and composite connection $Q_{1}$, a complex three-form field strength $G_{3}$ and a self-dual five-form $F_{5}=* F_{5}$. From a stringy point of view, the one-forms $P$ and $Q$ are related to the dilaton and RR axion, while $G_{3}$ contains both NSNS and RR (real) three-forms. The exact map is spelled out in appendix B.

The IIB supergravity fields satisfy the Bianchi identities:

$$
\begin{aligned}
d P & =2 i Q \wedge P, \\
d Q & =-i P \wedge \bar{P}, \\
d G_{3} & =i Q \wedge G_{3}-P \wedge \bar{G}_{3} .
\end{aligned}
$$

These are automatically satisfied if we introduce a complex scalar $B$ and a complex two form $C_{2}$ as:

$$
\begin{aligned}
P & =\left(1-|B|^{2}\right)^{-1} d B, \\
Q & =\left(1-|B|^{2}\right)^{-1} \Im[B d \bar{B}], \\
G_{3} & =\left(1-|B|^{2}\right)^{-\frac{1}{2}}\left(d C_{2}-B d \bar{C}_{2}\right) .
\end{aligned}
$$


The equations of motion are:

$$
\begin{aligned}
(d-2 i Q) \wedge * P & =-\frac{1}{4} G_{3} \wedge * G_{3}, \\
(d-i Q) \wedge * G_{3} & =P \wedge * \bar{G}_{3}-4 i G_{3} \wedge * F_{5}, \\
d * F_{5} & =\frac{i}{8} G_{3} \wedge \bar{G}_{3},
\end{aligned}
$$

along with the Einstein equation

$$
\begin{aligned}
R_{I J}= & P_{I} \bar{P}_{J}+\bar{P}_{I} P_{J}+\frac{1}{6} F_{I K L M N} F_{J}{ }^{K L M N} \\
& +\frac{1}{8}\left(G_{I K L} \bar{G}_{J}^{K L}+\bar{G}_{I K L} G_{J}{ }^{K L}\right)-\frac{1}{48} g_{I J} G_{K L M} \bar{G}^{K L M} .
\end{aligned}
$$

Note that the five-form equation can equally well be viewed as a Bianchi identity, $d F_{5}=$ $\frac{i}{8} G_{3} \wedge \bar{G}_{3}$, which may be solved by taking $F_{5}=d C_{4}+\frac{i}{8} \Im\left[C_{2} \wedge d \bar{C}_{2}\right]$. However, as in other IIB reductions, it is more convenient to make the ansatz on the field strength, and not the potential, in which case the equation of motion (2.3c) will need to be verified.

\subsection{A family of warped $A d S_{6}$ solutions}

A large family of $1 / 2$ BPS solutions to IIB supergravity of the form $A d S_{6} \times S^{2}$ warped over a 2D Riemann surface $\Sigma$ (with complex coordinates $z, \bar{z}$ ) were found by a detailed analysis of the IIB supersymmetry equations in [14]. These solutions are (locally) completely determined by a pair of holomorphic functions $\mathcal{A}_{ \pm}(z)$ on the Riemann surface. Remarkably, there is no restriction on the pair of holomorphic functions in order to obtain a local supersymmetric solution. ${ }^{1}$ To obtain a well-behaved global solution, considerably more care is needed: $\mathcal{A}_{ \pm}$are allowed to be multi-valued as holomorphic sections of a holomorphic bundle over $\Sigma$ with structure group contained in $\mathrm{SU}(1,1) \times \mathbb{C}$; the boundaries and other global properties of $\Sigma$ are important in constructing such solutions [14-16, 27]. There has been much interest in these global solutions and interpreting them as near-horizon limits of $(p, q)$ 5-brane webs with additional 7-branes in the web [15, 16, 27-29]. (See also section 4.1 for more discussion regarding the holographic duals of these solutions.)

Here we briefly summarize the solutions of [14]. The starting point is a pair of holomorphic functions $\mathcal{A}_{ \pm}(z)$, from which we may define a holomorphic function $\mathcal{B}$ whose derivative is given by (using obvious notation $\partial=\partial_{z}$ ):

$$
\partial_{z} \mathcal{B}=\mathcal{A}_{+} \partial \mathcal{A}_{-}-\mathcal{A}_{-} \partial \mathcal{A}_{+}
$$

Of the undetermined integration constant in $\mathcal{B}$, only the real part is relevant. From $\mathcal{A}_{ \pm}$ and $\mathcal{B}$, we define

$$
\kappa_{ \pm}=\partial \mathcal{A}_{ \pm}, \quad \kappa^{2}=-\left|\kappa_{+}\right|^{2}+\left|\kappa_{-}\right|^{2}
$$

along with

$$
\mathcal{G}=\left|\mathcal{A}_{+}\right|^{2}-\left|\mathcal{A}_{-}\right|^{2}+\mathcal{B}+\overline{\mathcal{B}}
$$

\footnotetext{
${ }^{1} \mathrm{~A}$ different but presumably equivalent classification of these $A d S_{6}$ solutions was found in [26]. These solutions were given in terms of solutions to the spherically symmetric cylindrical Laplace equation instead of holomorphic functions.
} 
Note that $\kappa^{2}=-\partial \bar{\partial} G$. We also find it useful to introduce

$$
\mathcal{Y}=\frac{\kappa^{2} \mathcal{G}}{|\partial \mathcal{G}|^{2}}, \quad \hat{\mathcal{C}}=\frac{\kappa_{+} \bar{\partial} \mathcal{G}+\bar{\kappa}_{-} \partial \mathcal{G}}{\kappa^{2}} .
$$

The supersymmetric $A d S_{6}$ vacua of [14] are then given by the metric

$$
d s^{2}=f_{6}^{2} d s_{A d S_{6}}^{2}+f_{2}^{2} d s_{S^{2}}^{2}+4 \rho^{2} d z d \bar{z},
$$

with metric functions

$$
f_{6}^{2}=\frac{c_{6}^{2}}{\rho^{2}} \kappa^{2} \sqrt{\tilde{\mathcal{D}}}, \quad f_{2}^{2}=\frac{c_{6}^{2}}{9 \rho^{2}} \frac{\kappa^{2}}{\sqrt{\tilde{\mathcal{D}}}}, \quad \rho^{4}=\frac{c_{6}^{2}}{6} \frac{\kappa^{4} \sqrt{\tilde{\mathcal{D}}}}{\mathcal{G}}
$$

and matter fields

$$
\begin{aligned}
B & =\frac{\left(\mathcal{A}_{+}-\overline{\mathcal{A}}_{-}\right)-\hat{\mathcal{C}} / \sqrt{\tilde{\mathcal{D}}}}{\left(\overline{\mathcal{A}}_{+}-\mathcal{A}_{-}\right)+\hat{\hat{\mathcal{C}}} / \sqrt{\tilde{\mathcal{D}}}} \\
C_{2} & =\frac{2 i c_{6}}{9}\left[\frac{\hat{\mathcal{C}}}{\tilde{\mathcal{D}}}-3\left(\overline{\mathcal{A}}_{-}+\mathcal{A}_{+}\right)\right] \operatorname{vol}\left(S^{2}\right), \\
F_{5} & =0
\end{aligned}
$$

Here $c_{6}$ is a non-vanishing constant, and we have furthermore introduced the recurring factor

$$
\tilde{\mathcal{D}}=1+\frac{2}{3 \mathcal{Y}}
$$

which will play an important role in the generalization to the full KK reduction below.

Finally, note that there are in fact two branches of solutions, the first with

$$
\kappa^{2} \geq 0, \quad \mathcal{G} \geq 0,
$$

and the second with both quantities non-positive. (These two branches of solutions are mapped into each other by complex conjugation [14].) Of course, the metric functions $f_{2}^{2}, f_{6}^{2}$ and $\rho^{2}$ must all be positive. This will be the case provided we take the positive square-root $(\sqrt{\tilde{\mathcal{D}}}>0)$ on the first branch and the negative square-root $(\sqrt{\tilde{\mathcal{D}}}<0)$ on the second branch.

\section{$3 \quad 6 \mathrm{D}$ supergravity and the reduction ansatz}

The existence of a family of $A d S_{6}$ solutions suggests the possibility of a complete non-linear Kaluza-Klein reduction of IIB supergravity to six-dimensional $F(4)$ gauged supergravity. Here we first present a brief overview of the six-dimensional theory, and then turn to the full reduction ansatz generalizing the vacuum solution discussed above in section 2.2. 


\section{$3.1 \quad F(4)$ gauged supergravity}

Romans' six-dimensional $F(4)$ gauged supergravity [3] is a non-chiral theory with 16 real supercharges. The bosonic fields consist of a metric $\tilde{g}_{\mu \nu}$, a real scalar $\tilde{\phi}$, an Abelian twoform $\tilde{F}_{2}$ and three-form $\tilde{F}_{3}$, and three $\mathrm{SU}(2)$ gauge two-forms $\tilde{F}^{i}$. The bosonic Lagrangian may be written in a form notation $[3,17]$ as

$$
\begin{aligned}
\mathcal{L}= & R *_{6} \mathbb{1}-4 \frac{*_{6} d X \wedge d X}{X^{2}}-\tilde{g}^{2}\left(\frac{2}{9} X^{-6}-\frac{8}{3} X^{-2}-2 X^{2}\right) *_{6} 1 \\
& -\frac{1}{2} X^{4} *_{6} \tilde{F}_{3} \wedge \tilde{F}_{3}-\frac{1}{2} X^{-2}\left(*_{6} \tilde{F}_{2} \wedge \tilde{F}_{2}+\frac{1}{\tilde{g}^{2}} *_{6} \tilde{F}^{i} \wedge \tilde{F}^{i}\right) \\
& -\tilde{A}_{2} \wedge\left(\frac{1}{2} d \tilde{A}_{1} \wedge d \tilde{A}_{1}+\frac{1}{3} \tilde{g} \tilde{A}_{2} \wedge d \tilde{A}_{1}+\frac{2}{27} \tilde{g}^{2} \tilde{A}_{2} \wedge \tilde{A}_{2}+\frac{1}{2 \tilde{g}^{2}} \tilde{F}^{i} \wedge \tilde{F}^{i}\right),
\end{aligned}
$$

where $X=e^{-\frac{1}{2 \sqrt{2}} \tilde{\phi}}$, and the field strengths are given in terms of the gauge potentials by

$$
\begin{aligned}
\tilde{F}_{3} & =d \tilde{A}_{2}, \\
\tilde{F}_{2} & =d \tilde{A}_{1}+\frac{2}{3} \tilde{g} \tilde{A}_{2}, \\
\tilde{F}^{i} & =d \tilde{A}^{i}+\frac{1}{2} \epsilon_{i j k} \tilde{A}^{j} \wedge \tilde{A}^{k} .
\end{aligned}
$$

The equations of motion corresponding to this Lagrangian are given by

$$
\begin{aligned}
d\left(X^{4} *_{6} \tilde{F}_{3}\right)= & -\frac{1}{2} \tilde{F}_{2} \wedge \tilde{F}_{2}-\frac{1}{2 \tilde{g}^{2}} \tilde{F}^{i} \wedge \tilde{F}^{i}-\frac{2}{3} \tilde{g} X^{-2} *_{6} \tilde{F}_{2}, \\
d\left(X^{-2} *_{6} \tilde{F}_{2}\right)= & -\tilde{F}_{2} \wedge \tilde{F}_{3}, \\
D\left(X^{-2} *_{6} \tilde{F}^{i}\right)= & -\tilde{F}_{3} \wedge \tilde{F}^{i}, \\
d\left(X^{-1} *_{6} d X\right)= & \frac{1}{8} X^{-2}\left(*_{6} \tilde{F}_{2} \wedge \tilde{F}_{2}+\frac{1}{\tilde{g}^{2}} *_{6} \tilde{F}^{i} \wedge \tilde{F}^{i}\right)-\frac{1}{4} X^{4} *_{6} \tilde{F}_{3} \wedge \tilde{F}_{3} \\
& +\tilde{g}^{2}\left(\frac{1}{6} X^{-6}-\frac{2}{3} X^{-2}+\frac{1}{2} X^{2}\right) *_{6} \mathbb{1},
\end{aligned}
$$

along with the Einstein equation

$$
\begin{aligned}
\tilde{R}_{\mu \nu}= & 4 X^{-2} \partial_{\mu} X \partial_{\nu} X+\tilde{g}^{2}\left(\frac{1}{18} X^{-6}-\frac{2}{3} X^{-2}-\frac{1}{2} X^{2}\right) g_{\mu \nu}+\frac{1}{4} X^{4}\left(\left(\tilde{F}_{3}\right)_{\mu \nu}^{2}-\frac{1}{6} g_{\mu \nu}\left(\tilde{F}_{3}\right)^{2}\right) \\
& +\frac{1}{2} X^{-2}\left(\left(\tilde{F}_{2}\right)_{\mu \nu}^{2}-\frac{1}{8} g_{\mu \nu}\left(\tilde{F}_{2}\right)^{2}\right)+\frac{1}{2 \tilde{g}^{2}} X^{-2}\left(\left(\tilde{F}^{i}\right)_{\mu \nu}^{2}-\frac{1}{8} g_{\mu \nu}\left(\tilde{F}^{i}\right)^{2}\right) .
\end{aligned}
$$

In the above, the $\mathrm{SU}(2)$ gauge covariant derivative $D$ is defined by

$$
D \tilde{F}^{i}=d \tilde{F}^{i}+\epsilon_{i j k} \tilde{A}^{j} \wedge \tilde{F}^{k} .
$$

This $F(4)$ gauged supergravity theory admits a supersymmetric $A d S_{6}$ vacuum with $\tilde{F}_{2}=\tilde{F}_{3}=\tilde{F}^{i}=0$ and $X=1$ that preserves all 16 of the supersymmetries [3]. There is also a non-supersymmetric $A d S_{6}$ vacuum with $X=3^{-1 / 4}$. Note that to get a unit radius $A d S_{6}$, we must choose:

$$
\tilde{g}=\frac{3}{\sqrt{2}} .
$$

We will make this choice from here on. 


\subsection{Generalized reduction ansatz}

The $F(4)$ gauged supergravity theory was obtained from a warped $S^{4}$ reduction of massive IIA supergravity in [17], and more recently from a reduction of IIB supergravity over an $S^{2}$ and a Riemann surface in [18]. In both cases, the supersymmetric $A d S_{6}$ vacua uplifted to $10 \mathrm{D}$ are $1 / 2$ BPS solutions of either IIA or IIB supergravity.

We now present our main result, which is a consistent truncation of type IIB supergravity to $6 \mathrm{D} F(4)$ gauged supergravity generalizing the reduction of $[17,18]$. We start with the metric

$$
d s^{2}=f_{6}^{2} d s_{6}^{2}+f_{2}^{2} d s_{\tilde{S}^{2}}^{2}+4 \rho^{2} d z d \bar{z}
$$

where

$$
f_{6}^{2}=\frac{c_{6}^{2}}{\rho^{2}} \kappa^{2} \sqrt{\mathcal{D}}, \quad f_{2}^{2}=\frac{c_{6}^{2}}{9 \rho^{2}} \frac{\kappa^{2} X^{2}}{\sqrt{\mathcal{D}}}, \quad \rho^{4}=\frac{c_{6}^{2}}{6} \frac{\kappa^{4} X^{2} \sqrt{\mathcal{D}}}{\mathcal{G}} .
$$

The matter fields are given by

$$
\begin{aligned}
B & =\frac{\left(\mathcal{A}_{+}-\overline{\mathcal{A}}_{-}\right)-\hat{\mathcal{C}} X^{2} / \sqrt{\mathcal{D}}}{\left(\overline{\mathcal{A}}_{+}-\mathcal{A}_{-}\right)+\hat{\hat{\mathcal{C}}} X^{2} / \sqrt{\mathcal{D}}} \\
C_{2} & =\frac{2 i c_{6}}{9}\left[\frac{\hat{\mathcal{C}}}{\mathcal{D}}-3\left(\overline{\mathcal{A}}_{-}+\mathcal{A}_{+}\right)\right] \operatorname{vol}\left(\tilde{S}^{2}\right)-\sqrt{2} c_{6}\left(\overline{\mathcal{A}}_{-}-\mathcal{A}_{+}\right) \tilde{F}_{2}+\frac{2 i c_{6}}{3}\left(\overline{\mathcal{A}}_{-}+\mathcal{A}_{+}\right) \mu^{i} \tilde{F}^{i}, \\
F_{5} & =G_{5}+* G_{5},
\end{aligned}
$$

with

$$
G_{5}=c_{6}^{2}\left[\frac{i \kappa^{2} X^{4}}{2}\left(*_{6} \tilde{F}_{3}\right) \wedge d z \wedge d \bar{z}+\frac{1}{2 \sqrt{2} X^{2}}\left(*_{6} \tilde{F}_{2}\right) \wedge *_{2} d \mathcal{G}-\frac{1}{6 X^{2}}\left(*_{6} \tilde{F}^{i}\right) \wedge D\left(\mu^{i} \mathcal{G}\right)\right] .
$$

It is also useful to give the expression for $* G_{5}$ :

$$
\begin{aligned}
* G_{5}=c_{6}^{2}[ & \left(\frac{\mathcal{G} X^{4}}{6 \mathcal{D}} \tilde{F}_{3}+\frac{\sqrt{2}}{36 \mathcal{D}} \tilde{F}_{2} \wedge d \mathcal{G}+\frac{1}{54 \mathcal{D}} \mu^{i} \tilde{F}^{i} \wedge *_{2} d \mathcal{G}\right) \wedge \operatorname{vol}\left(\tilde{S}^{2}\right) \\
& \left.+\frac{i \kappa^{2}}{18} \tilde{F}^{i} \wedge *_{2} D \mu^{i} \wedge d z \wedge d \bar{z}\right] .
\end{aligned}
$$

Here $\mathcal{D}$ is defined as

$$
\mathcal{D}=X^{4}+\frac{2}{3 \mathcal{Y}}
$$

which generalizes (2.12) in the presence of a non-trivial scalar. Note that $d s_{\tilde{S}^{2}}^{2}$ and $\operatorname{vol}\left(\tilde{S}^{2}\right)$ have been used instead of $d s_{S^{2}}^{2}$ and $\operatorname{vol}\left(S^{2}\right)$ since the $\mathrm{SU}(2)$ isometry of the $S^{2}$ is gauged by the $\tilde{A}^{i}$ fields. (See appendix A for more information.) The remaining definitions, (2.5) through (2.8), are unchanged.

Our reduction ansatz thus provides a consistent truncation of IIB supergravity on an $S^{2}$ and warped over a Riemann surface $\Sigma$ to $6 \mathrm{D} F(4)$ gauged supergravity. In fact, our ansatz contains a family of such truncations - one for each pair of holomorphic functions $\mathcal{A}_{ \pm}$on the Riemann surface. This fully generalizes and contains the supersymmetric $A d S_{6}$ solutions of [14] discussed in section 2.2. In particular, (3.8) and (3.9a) generalize the corresponding expressions (2.10) and (2.11a) to incorporate the scalar $X$, and (3.9b) 
and (3.9c) generalize (2.11b) and (2.11c) to incorporate the six-dimensional gauge fields. When $X=1$ (i.e. the scalar $\tilde{\phi}=0$ ) and the gauge fields are turned off, $\tilde{F}_{2}=\tilde{F}_{3}=\tilde{F}^{i}=0$, this reduction ansatz reduces as it must to that of [14].

We have explicitly checked that our ansatz (3.7)-(3.10) satisfies the 10D IIB equations of motion (2.3) if and only if the $6 \mathrm{D}$ fields $\tilde{g}_{\mu \nu}, \tilde{F}_{2}, \tilde{F}_{3}, \tilde{F}^{i}$ and $X$ satisfy the $6 \mathrm{D}$ equations of motion (3.3) and (3.4). In particular, the IIB axi-dilaton equation of motion (2.3a) is equivalent to the $6 \mathrm{D}$ scalar equation of motion (3.3d), the IIB three-form equation $(2.3 \mathrm{~b})$ is equivalent to $(3.3 \mathrm{a}),(3.3 \mathrm{~b}),(3.3 \mathrm{c})$, and $(3.3 \mathrm{~d})$, and the self-dual five-form equation $(2.3 \mathrm{c})$ is equivalent to (3.3a), (3.3b), and (3.3c). Finally, the IIB Einstein equation, (2.4), is satisfied if and only if (3.3c), (3.3d) and the 6D Einstein equation (3.4) are satisfied. For more details of these calculations, see appendix C.

Our ansatz thus gives a family of consistent truncations of 10D IIB supergravity to 6D $F(4)$ gauged supergravity, in the sense that any solution of the $6 \mathrm{D}$ theory can be uplifted to a family of $10 \mathrm{D}$ solutions using (3.7)-(3.10). We stress once more that this ansatz is consistent for any pair of holomorphic functions $\mathcal{A}_{ \pm}$, at least up to regularity conditions of the $A d S_{6}$ vacuum.

\section{Discussion}

The reduction ansatz (3.7)-(3.10) generalizes the IIB reduction of [18], corresponding to the $A d S_{6}$ background of [19], to encompass the complete family of $A d S_{6}$ backgrounds of [14]. In fact, our method for finding this generalized ansatz was to translate the specific reduction of [18] into the language of the holomorphic functions $\mathcal{A}_{ \pm}$of [14]. For example, starting with the string frame metric embedding of [18]

$$
d \hat{s}^{2}=X^{-\frac{1}{2}} s^{-\frac{1}{3}} \Delta^{\frac{1}{2}}\left[d s_{6}^{2}+2 \tilde{g}^{-2} X^{2} d \xi^{2}\right]+e^{-2 A} d r^{2}+\frac{r^{2} e^{2 A}}{r^{2}+e^{4 A}} d s_{\tilde{S}^{2}}^{2},
$$

with

$$
e^{A}=\frac{1}{\sqrt{2} \tilde{g}} s^{-\frac{1}{6}} c X^{-\frac{3}{4}} \Delta^{-\frac{1}{4}}, \quad \Delta=X c^{2}+X^{-3} s^{2}, \quad s=\sin \xi, \quad c=\cos \xi,
$$

we deduce the coordinate transformation on the Riemann surface $\Sigma$ mapping $\{\xi, r\} \rightarrow$ $\{z, \bar{z}\}$

$$
z=z_{1}+i z_{2}, \quad z_{1}=\frac{2}{3} \tilde{g}^{2} r, \quad z_{2}=\sin ^{2 / 3} \xi
$$

This allows us to rewrite (4.1) as

$$
d \hat{s}^{2}=X^{-\frac{1}{2}} s^{-\frac{1}{3}} \Delta^{\frac{1}{2}} d s_{6}^{2}+\frac{r^{2} e^{2 A}}{r^{2}+e^{4 A}} d s_{\tilde{S}^{2}}^{2}+\frac{9}{4 \tilde{g}^{4}} e^{-2 A} d z d \bar{z} .
$$

Converting to the Einstein frame and comparing with the $A d S_{6}$ metric (3.7) then yields the $X$-dependent metric functions (3.8), provided we identify

$$
\kappa^{2}=\frac{z_{1} z_{2}}{36 c_{6}^{2}}, \quad \mathcal{G}=\frac{\left(1-z_{2}^{3}\right) z_{1}}{54 c_{6}^{2}}, \quad|\partial \mathcal{G}|^{2}=\frac{z_{1}^{2} z_{2}^{4}+\frac{1}{9}\left(1-z_{2}^{3}\right)^{2}}{\left(36 c_{6}^{2}\right)^{2}},
$$

where we have additionally set $\tilde{g}=3 / \sqrt{2}$ as in (3.6). 
Working backwards from (4.5), it is not too difficult to deduce the form of the holomorphic functions $\mathcal{A}_{ \pm}$

$$
\mathcal{A}_{ \pm}(z)=\frac{1}{c_{6}}\left(\frac{1}{216} z^{3} \mp \frac{i}{4} z-\frac{i}{108}\right)
$$

along with the auxiliary function

$$
\mathcal{B}(z)=\frac{1}{c_{6}^{2}}\left(-\frac{i}{864} z^{4}+\frac{1}{216} z\right) .
$$

Given these functions, it is then possible to work out (3.9a) as the appropriate generalization of the IIB axi-dilaton reduction (2.11a) in the presence of a non-trivial scalar $X$. The remaining expressions are then for the complex three-form $G_{3}$ and self-dual five-form $F_{5}$. These take a bit more effort, but can be obtained by translating the particular ansatz of [18] into the $\mathrm{SU}(1,1)$ Einstein frame and reexpressing the result in terms of the holomorphic functions $\mathcal{A}_{ \pm}$and their derived quantities such as $\mathcal{G}$ and $\kappa_{ \pm}$. Finally, as it was not guaranteed that this construction would be a consistent truncation, it was essential to check the IIB equations of motion and verify that they were equivalent to the $F(4)$ gauged supergravity equations of motion - and in particular, that they did not impose any extra conditions on the functions $\mathcal{A}_{ \pm}$. Additional details of these checks are presented in appendix C.

\subsection{Holography}

Finally, we conclude with a few remarks about $A d S_{6} / C F T_{5}$ holography. From a 10D point of view, we may use the supersymmetric $A d S_{6}$ solutions of [14-16] discussed in section 2.2, see e.g. [27-32]. These efforts have been limited to the supersymmetric $A d S_{6}$ vacua as the extension of these solutions to include non-BPS excitations were not known until now.

Alternatively, one can approach $6 \mathrm{D} / 5 \mathrm{D}$ holography in supergravity from the $6 \mathrm{D}$ perspective; efforts using the 6D F(4) supergravity discussed in section 3.1 include [33-44]. The 6D supergravity is then the effective theory that describes (a consistent truncation of the set of) excitations around the $A d S_{6}$ vacuum. However, without an understanding of the uplift of these $6 \mathrm{D}$ solutions to $10 \mathrm{D}$, a microscopic understanding of the CFT described by the $A d S_{6}$ vacuum and its excitations was lacking.

Our reduction ansatz (3.7)-(3.10) provides a key link between the 10D and 6D approaches above. In the 10D approach, it provides a way to include (non-BPS) excitations to the family of $A d S_{6}$ vacua. From the $6 \mathrm{D}$ perspective, it gives a way to understand the microscopic dual 5D CFT through the brane picture obtained by the $10 \mathrm{D}$ uplift of the $6 \mathrm{D}$ solutions. For example, the fact that our uplift is independent of the choice of holomorphic functions $\mathcal{A}_{ \pm}$shows that it is perhaps not particularly surprising that the massive spin-2 excitations around the supersymmetric $A d S_{6}$ vacua found in [31] are universally present for any $\mathcal{A}_{ \pm}$.

It would be interesting to extend the ansatz (3.7)-(3.10) further to include more fields in the truncation from 10D. For example, one could consider coupling vector multiplets to the 6D F(4) theory; such an ansatz would then provide a 10D uplift of the 6D Janus solutions found in [42], which should correspond to mass deformations of the dual 5D SCFTs. It is not obvious, however, whether the consistent truncation obtained here can be generalized to include the addition of matter multiplets, as non-linear Kaluza-Klein consistency often requires a delicate balance between internal harmonics unless a symmetry argument can 
be made $[45,46]$, which does not appear to be the case for the Janus solutions. A possibly more fruitful approach would be to consider instead gauge fields that arise from D3-branes wrapping 3-cycles [29], as they are not subject to the standard constraints of Kaluza-Klein consistency.

\section{Acknowledgments}

This project was initiated following discussions with C.F. Uhlemann at the 2018 USU Workshop on Strings and Black Holes. We wish to thank C.F. Uhlemann for enlightening discussions and E. O Colgain and O. Varela for useful comments. This work was supported in part by the U.S. Department of Energy under grant DE-SC0007859.

\section{A Conventions}

For $10 \mathrm{D}$ indices, we use $K, L, M, \ldots$, and for $6 \mathrm{D}$ indices we use $\mu, \nu, \rho, \ldots$. We work in both six and ten dimensions with a mostly plus signature. In particular, the $F(4)$ gauged supergravity Lagrangian given in (3.1) has been converted from the original mostly minus signature of [3].

The complex coordinates on the 2D Riemann surface $\Sigma$ are $z, \bar{z}$. With respect to these coordinates, we define:

$$
*_{2} d z=i d z \text {. }
$$

On the two-sphere $S^{2}$, we can use (three) constrained coordinates $\mu^{i}$ which satisfy $\sum_{i=1}^{3} \mu^{i} \mu^{i}=1$. The metric and the volume form on the $S^{2}$ are then:

$$
d s_{S^{2}}^{2}=\sum_{i=1}^{3} d \mu^{i} d \mu^{i}, \quad \operatorname{vol}\left(S^{2}\right)=\frac{1}{2} \epsilon_{i j k} \mu^{i} d \mu^{j} \wedge d \mu^{k},
$$

respectively. The $\mathrm{SU}(2)$ gauge-covariant derivative $D$ is defined by:

$$
D \tilde{F}^{i}=d \tilde{F}^{i}+\epsilon_{i j k} \tilde{A}^{j} \wedge \tilde{F}^{k} .
$$

The natural metric and the volume form on the $S^{2}$ when the $\mathrm{SU}(2)$ isometries are gauged by the $\tilde{F}^{i}$ gauge fields are then:

$$
d s_{\tilde{S}^{2}}^{2}=\sum_{i=1}^{3} D \mu^{i} D \mu^{i}, \quad \operatorname{vol}\left(\tilde{S}^{2}\right)=\frac{1}{2} \epsilon_{i j k} \mu^{i} D \mu^{j} \wedge D \mu^{k},
$$

respectively. We also define:

$$
*_{2} D \mu^{i}=\epsilon_{i j k} \mu^{j} D \mu^{k} .
$$

Even though the same symbol $*_{2}$ is used for Hodge duality on the $S^{2}$ and $\Sigma$, it should be clear which is meant by the context it is used in. Note that an explicit form of the $\mu^{i}$ coordinates could be:

$$
\mu^{1}=\sin \theta \sin \phi, \quad \mu^{2}=\sin \theta \cos \phi, \quad \mu^{3}=-\cos \theta,
$$

so that the ungauged metric is the usual $d s_{S^{2}}^{2}=d \theta^{2}+\sin ^{2} \theta d \phi^{2}$. 
Our conventions for Hodge duality are given by:

$$
*\left(d x^{\mu_{1}} \wedge \cdots \wedge d x^{\mu_{r}}\right)=\frac{\sqrt{|g|}}{(D-r) !} \epsilon_{\nu_{1} \cdots \nu_{D-r}}^{\mu_{1} \cdots \mu_{r}} d x^{\nu_{1}} \wedge \cdots \wedge d x^{\nu_{D-r}}
$$

where $D$ is the dimension. The relation between 10D and 6D Hodge duality is given by

$$
\epsilon_{\mu_{1} \cdots \mu_{6} \alpha \beta i j}^{(10 D)}=\epsilon_{\mu_{1} \cdots \mu_{6}}^{(6 D)} \epsilon_{\alpha \beta} \epsilon_{i j}=1
$$

where $\alpha, \beta$ are orthonormal coordinates for the $\tilde{S}_{2}$ and $i, j$ are real coordinates on $\Sigma$. The orientation on $\tilde{S}_{2}$ and $\Sigma$ are given by (A.5) and (A.1), respectively.

\section{B Mapping between different 10D IIB conventions}

Type IIB supergravity in 10D is often formulated in terms of the (real) axion $\hat{C}_{0}$ with field strength $\hat{F}_{1}=d \hat{C}_{0}$, dilaton $\hat{\phi}$; the RR and NSNS three-form field strengths $\hat{F}_{3}$ and $\hat{H}_{3}{ }^{2}$ The Bianchi identities of these fields are:

$$
\begin{aligned}
& 0=d \hat{F}_{1}, \\
& 0=d \hat{F}_{3}-\hat{H}_{3} \wedge \hat{F}_{1}, \\
& 0=d \hat{H}_{3},
\end{aligned}
$$

of which the last two are automatically satisfied when we introduce the (real) two-form gauge potentials $\hat{C}_{2}$ and $\hat{B}_{2}$ as:

$$
\begin{aligned}
\hat{F}_{3} & =d \hat{C}_{2}-\hat{H}_{3} \hat{C}_{0}, \\
\hat{H}_{3} & =d \hat{B}_{2} .
\end{aligned}
$$

The map between the complex fields $B, C_{2}$ used in section 2 and the real fields $\hat{C}_{0}, \hat{\phi}, \hat{C}_{2}$, $\hat{B}_{2}$ is:

$$
\begin{aligned}
B & =\frac{1+i \tau}{1-i \tau}, \\
C_{2} & =\hat{B}_{2}+i \hat{C}_{2} .
\end{aligned} \quad \tau=\hat{C}_{0}+i e^{-\hat{\phi}}
$$

The string frame metric $\hat{g}_{M N}$ is related to the Einstein frame metric (used in section 2) as:

$$
\hat{g}_{M N}=e^{\hat{\phi} / 2} g_{M N}
$$

\footnotetext{
${ }^{2}$ We use hats to denote string frame fields, while un-hatted fields denote Einstein frame fields as used in section 2 .
} 
The equations of motion for the real 10D fields in string frame (including the self-dual five-form $F_{5}{ }^{3}$ ) are then given by (with $\hat{*}$ denoting the string frame Hodge dual):

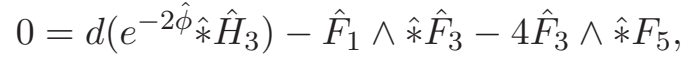

$$
\begin{aligned}
& 0=d \hat{*} \hat{F}_{1}+\hat{H}_{3} \wedge \hat{*} \hat{F}_{3}, \\
& 0=d \hat{*} \hat{F}_{3}+4 \hat{H}_{3} \wedge \hat{*} F_{5} \text {, } \\
& 0=\hat{R}+4 \hat{\nabla}^{2} \hat{\phi}-4(\partial \hat{\phi})^{2}-\frac{1}{12}\left(\hat{H}_{3}\right)^{2}, \\
& 0=\hat{R}_{M N}+2 \hat{\nabla}_{M} \hat{\nabla}_{N} \hat{\phi}-\frac{1}{4}\left(\hat{H}_{3}\right)_{M N}^{2} \\
& -e^{2 \hat{\phi}}\left[\frac{1}{2}\left(\hat{F}_{1}\right)_{M N}^{2}+\frac{1}{4}\left(\hat{F}_{3}\right)_{M N}^{2}+\frac{1}{6}\left(F_{5}\right)_{M N}^{2}-\frac{1}{4} \hat{g}_{M N}\left(\hat{F}_{1}^{2}+\frac{1}{6} \hat{F}_{3}^{2}\right)\right], \\
& 0=d F_{5}-\frac{1}{4} \hat{H}_{3} \wedge \hat{F}_{3},
\end{aligned}
$$

with the obvious notation of e.g.:

$$
\left(\hat{H}_{3}\right)_{M N}^{2}=\left(\hat{H}_{3}\right)_{M}^{K L}\left(\hat{H}_{3}\right)_{N K L}
$$

\section{Checking the 10D EOMs}

We have verified that the 10D equations of motion (2.3) and (2.4) on the general reduction ansatz (3.7)-(3.10) are equivalent to the 6D equations of motion (3.3) and (3.4). Here we give some details of this tedious but straightforward calculation.

The basic strategy is to write the forms (and their wedge products, Hodge duals, and exterior derivatives) as much as possible in terms of the derived quantities $d \mathcal{G}, d(\mathcal{G} / \mathcal{Y})$ and their 2D Hodge duals on the Riemann surface $*_{2} d \mathcal{G}, *_{2} d(\mathcal{G} / \mathcal{Y})$. Then, we can use some simple identities that are valid on the Riemann surface, such as:

$$
\begin{aligned}
d *_{2} d \mathcal{G} & =2 i \kappa^{2} d z \wedge d \bar{z} \\
d \mathcal{G} \wedge *_{2} d \mathcal{G} & =-2 i \kappa^{2} \frac{\mathcal{G}}{\mathcal{Y}} d z \wedge d \bar{z} \\
\left(\frac{\mathcal{G}}{\mathcal{Y}}\right) d *_{2} d\left(\frac{\mathcal{G}}{\mathcal{Y}}\right) & =3 d \mathcal{G} \wedge *_{2} d \mathcal{G}+4 d \mathcal{G} \wedge *_{2} d\left(\frac{\mathcal{G}}{\mathcal{Y}}\right)+2 d\left(\frac{\mathcal{G}}{\mathcal{Y}}\right) \wedge *_{2} d\left(\frac{\mathcal{G}}{\mathcal{Y}}\right)
\end{aligned}
$$

One also needs to utilize a number of identities that the coordinates $\mu^{i}$ on the $S^{2}$ satisfy together with the gauge fields $\tilde{F}^{i}$, such as:

$$
\begin{aligned}
d \operatorname{vol}\left(\tilde{S}^{2}\right) & =d\left(\mu^{i} \tilde{F}^{i}\right)=\tilde{F}^{i} \wedge D \mu^{i}, \\
D \mu^{i} \wedge \operatorname{vol}\left(\tilde{S}^{2}\right) & =0, \\
\epsilon_{i j k} D \mu^{j} \wedge D \mu^{k} & =2 \mu^{i} \operatorname{vol}\left(\tilde{S}^{2}\right), \\
d\left(\tilde{F}^{i} \wedge *_{2} D \mu^{i}\right) & =2 \mu^{i} \tilde{F}^{i} \wedge \operatorname{vol}\left(\tilde{S}^{2}\right)+\tilde{F}^{i} \wedge \tilde{F}^{i}-\mu^{i} \tilde{F}^{i} \wedge \mu^{j} \tilde{F}^{j} .
\end{aligned}
$$

\footnotetext{
${ }^{3}$ Often a different normalization for $F_{5}$ is used, e.g. $\hat{F}_{5}=4 F_{5}$ in [18].
} 
The IIB five-form and metric are given directly in (3.9c), (3.10), (3.7) and (3.8). For the remaining fields $P, Q$ and $G_{3}$, we first note that the IIB equations of motion are invariant under the $\mathrm{U}(1)$ transformation:

$$
P \rightarrow e^{2 i \theta} P, \quad Q \rightarrow Q+d \theta, \quad G_{3} \rightarrow e^{i \theta} G_{3},
$$

Inserting the expressions (3.9a) and (3.9b) for $B$ and $C_{2}$ into the expressions (2.2), and furthermore performing a $\mathrm{U}(1)$ transformation with phase

$$
e^{i \theta}=\frac{\left(\mathcal{A}_{+}-\overline{\mathcal{A}}_{-}\right)+\hat{C} X^{2} / \sqrt{D}}{\left|\left(\mathcal{A}_{+}-\overline{\mathcal{A}}_{-}\right)+\hat{C} X^{2} / \sqrt{D}\right|}
$$

then gives

$$
\begin{aligned}
& P=-\frac{2}{3 \mathcal{Y D}} \frac{d X}{X}+ \frac{\mathcal{Y} \sqrt{\mathcal{D}}}{2 \mathcal{G} X^{2}}\left[i *_{2} \mathcal{J}-\frac{X^{2}}{\sqrt{\mathcal{D}}} \mathcal{K}\right] \\
& Q=\frac{\mathcal{Y} \sqrt{\mathcal{D}}}{2 \mathcal{G} X^{2}} *_{2} \mathcal{L}, \\
& G_{3}=\left(\frac{\mathcal{Y} \sqrt{\mathcal{D}}}{\mathcal{G} X^{2}}\right)^{\frac{1}{2}}[\left(-\frac{8 i c_{6} \mathcal{G} X^{3}}{9 \mathcal{Y} \mathcal{D}^{2}} d X+\frac{c_{6}}{3}\left(i \mathcal{M}+\frac{X^{2}}{\sqrt{\mathcal{D}}} *_{2} \mathcal{N}\right)\right) \wedge \operatorname{vol}\left(\tilde{S}^{2}\right) \\
&+2 c_{6} \frac{\mathcal{G} X^{2}}{\mathcal{Y} \sqrt{\mathcal{D}}} \tilde{F}_{3}-\frac{c_{6}}{\sqrt{2}} \tilde{F}_{2} \wedge\left(i *_{2} d \mathcal{G}+\frac{X^{2}}{\sqrt{\mathcal{D}}} d \mathcal{G}\right) \\
&+\frac{c_{6}}{3}\left(\frac{2 i}{3} \frac{\mathcal{G}}{\mathcal{Y} \mathcal{D}} \tilde{F}^{i} \wedge D \mu^{i}+\mu^{i} \tilde{F}^{i} \wedge\left(i d \mathcal{G}-\frac{X^{2}}{\left.\left.\left.\sqrt{\mathcal{D}} *_{2} d \mathcal{G}\right)\right)\right],}\right.\right.
\end{aligned}
$$

where we have defined the shorthand one-forms $\mathcal{J}, \mathcal{K}, \mathcal{L}, \mathcal{M}, \mathcal{N}$ as:

$$
\begin{aligned}
\mathcal{J} & =\left(1-\frac{1}{\mathcal{Y D}}\right) d \mathcal{G}+\left(1-\frac{2}{3 \mathcal{Y D}}\right) d\left(\frac{\mathcal{G}}{\mathcal{Y}}\right) \\
\mathcal{K} & =\left(1+\frac{1}{3 \mathcal{Y}^{2} \mathcal{D}}\right) d \mathcal{G}+\left(1-\frac{1}{3 \mathcal{Y D}}\right) d\left(\frac{\mathcal{G}}{\mathcal{Y}}\right) \\
\mathcal{L} & =\left(2-\frac{1}{\mathcal{Y D}}\right) d \mathcal{G}+\left(1-\frac{2}{3 \mathcal{Y D}}\right) d\left(\frac{\mathcal{G}}{\mathcal{Y}}\right), \\
\mathcal{M} & =\left(\frac{4}{9 \mathcal{Y}^{2} \mathcal{D}^{2}}+\frac{1}{3 \mathcal{D}}-1\right) d \mathcal{G}+\frac{2}{3 \mathcal{D}}\left(1-\frac{2}{3 \mathcal{Y D}}\right) d\left(\frac{\mathcal{G}}{\mathcal{Y}}\right), \\
\mathcal{N} & =\left(1+\frac{1}{\mathcal{D}}\right) d \mathcal{G}+\frac{2}{3 \mathcal{D}} d\left(\frac{\mathcal{G}}{\mathcal{Y}}\right) .
\end{aligned}
$$

Now it is just a matter of using the above expressions to check the IIB equations of motion. For example, using the expressions (C.5) in the axi-dilaton equation of motion, (2.3a), gives

$$
\begin{aligned}
0=-\frac{8 i c_{6}^{4} \kappa^{2} \mathcal{G}}{9 \mathcal{Y D}}[ & d\left(X^{-1} *_{6} d X\right)-\frac{1}{4} X^{4}\left(\tilde{F}_{3} \wedge *_{6} \tilde{F}_{3}\right)-\frac{1}{8} X^{-2}\left(\tilde{F}_{2} \wedge *_{6} \tilde{F}_{2}+\frac{2}{9} \tilde{F}^{i} \wedge *_{6} \tilde{F}^{i}\right) \\
& \left.-\frac{9}{2}\left(\frac{1}{6} X^{-6}-\frac{2}{3} X^{-2}+\frac{1}{2} X^{2}\right)\left(*_{6} \mathbb{1}\right)\right] \wedge \operatorname{vol}\left(\tilde{S}^{2}\right) \wedge d z \wedge d \bar{z}
\end{aligned}
$$


which is equivalent to the $6 \mathrm{D}$ scalar equation of motion (3.3d). The other equations of motion for the form-fields (2.3b) and (2.3c) proceed similarly (although they are more involved).

Finally, the Einstein equations are perhaps the most tedious of all to check. In the end, the $(\mu \nu)$ components of the 10D Einstein equations reduce to the 6D Einstein equations (3.4) and scalar equation (3.3d) of motion. The $(z \bar{z})$ component of the Einstein equations reduces to the scalar equation (3.3d), as do the components with both legs on the $S^{2}$. Finally, the components with one leg in the $6 \mathrm{D}$ manifold and one leg on the $S^{2}$ reduces to the equation of motion (3.3c) for the $\mathrm{SU}(2)$ gauge field. (All other components of the Einstein equations reduce to identities.)

Open Access. This article is distributed under the terms of the Creative Commons Attribution License (CC-BY 4.0), which permits any use, distribution and reproduction in any medium, provided the original author(s) and source are credited.

\section{References}

[1] W. Nahm, Supersymmetries and their representations, Nucl. Phys. B 135 (1978) 149 [INSPIRE].

[2] S. Minwalla, Restrictions imposed by superconformal invariance on quantum field theories, Adv. Theor. Math. Phys. 2 (1998) 783 [hep-th/9712074] [INSPIRE].

[3] L.J. Romans, The F(4) gauged supergravity in six-dimensions, Nucl. Phys. B 269 (1986) 691 [INSPIRE].

[4] J.M. Figueroa-O'Farrill and G. Papadopoulos, Maximally supersymmetric solutions of ten-dimensional and eleven-dimensional supergravities, JHEP 03 (2003) 048 [hep-th/0211089] [INSPIRE].

[5] N. Seiberg, Five-dimensional SUSY field theories, nontrivial fixed points and string dynamics, Phys. Lett. B 388 (1996) 753 [hep-th/9608111] [INSPIRE].

[6] A. Brandhuber and Y. Oz, The D4-D8 brane system and five-dimensional fixed points, Phys. Lett. B 460 (1999) 307 [hep-th/9905148] [INSPIRE].

[7] O. Bergman and D. Rodriguez-Gomez, $5 D$ quivers and their $A d S_{6}$ duals, JHEP 07 (2012) 171 [arXiv: 1206.3503] [INSPIRE].

[8] A. Passias, A note on supersymmetric AdS $S_{6}$ solutions of massive type IIA supergravity, JHEP 01 (2013) 113 [arXiv:1209.3267] [inSPIRE].

[9] O. Aharony and A. Hanany, Branes, superpotentials and superconformal fixed points, Nucl. Phys. B 504 (1997) 239 [hep-th/9704170] [INSPIRE].

[10] O. Aharony, A. Hanany and B. Kol, Webs of $(p, q)$ five-branes, five-dimensional field theories and grid diagrams, JHEP 01 (1998) 002 [hep-th/9710116] [INSPIRE].

[11] O. DeWolfe, A. Hanany, A. Iqbal and E. Katz, Five-branes, seven-branes and five-dimensional $E_{n}$ field theories, JHEP 03 (1999) 006 [hep-th/9902179] [INSPIRE].

[12] F. Apruzzi, M. Fazzi, A. Passias, D. Rosa and A. Tomasiello, AdS $S_{6}$ solutions of type-II supergravity, JHEP 11 (2014) 099 [Erratum ibid. 05 (2015) 012] [arXiv:1406.0852] [INSPIRE]. 
[13] H. Kim, N. Kim and M. Suh, Supersymmetric AdS $S_{6}$ solutions of type IIB supergravity, Eur. Phys. J. C 75 (2015) 484 [arXiv:1506.05480] [InSPIRE].

[14] E. D'Hoker, M. Gutperle, A. Karch and C.F. Uhlemann, Warped $A d S_{6} \times S^{2}$ in type IIB supergravity I: local solutions, JHEP 08 (2016) 046 [arXiv: 1606.01254] [INSPIRE].

[15] E. D'Hoker, M. Gutperle and C.F. Uhlemann, Warped $A d S_{6} \times S^{2}$ in type IIB supergravity II: global solutions and five-brane webs, JHEP 05 (2017) 131 [arXiv: 1703.08186] [INSPIRE].

[16] E. D'Hoker, M. Gutperle and C.F. Uhlemann, Warped $A d S_{6} \times S^{2}$ in type IIB supergravity III: global solutions with seven-branes, JHEP 11 (2017) 200 [arXiv:1706.00433] [INSPIRE].

[17] M. Cvetič, H. Lü and C.N. Pope, Gauged six-dimensional supergravity from massive type IIA, Phys. Rev. Lett. 83 (1999) 5226 [hep-th/9906221] [INSPIRE].

[18] J. Jeong, O. Kelekci and E. O Colgain, An alternative IIB embedding of F(4) gauged supergravity, JHEP 05 (2013) 079 [arXiv:1302.2105] [INSPIRE].

[19] Y. Lozano, E. Ó Colgáin, D. Rodríguez-Gómez and K. Sfetsos, Supersymmetric AdS 6 via T duality, Phys. Rev. Lett. 110 (2013) 231601 [arXiv:1212.1043] [INSPIRE].

[20] M.J. Duff and C.N. Pope, Consistent truncations in Kaluza-Klein theories, Nucl. Phys. B 255 (1985) 355 [inSPIRE].

[21] J.P. Gauntlett and O. Varela, Consistent Kaluza-Klein reductions for general supersymmetric AdS solutions, Phys. Rev. D 76 (2007) 126007 [arXiv:0707.2315] [INSPIRE].

[22] E. Malek, Half-maximal supersymmetry from exceptional field theory, Fortsch. Phys. 65 (2017) 1700061 [arXiv:1707.00714] [INSPIRE].

[23] E. Malek, H. Samtleben and V. Vall Camell, Supersymmetric AdS $S_{7}$ and $A d S_{6}$ vacua and their minimal consistent truncations from exceptional field theory, arXiv:1808.05597 [INSPIRE].

[24] J.H. Schwarz, Covariant field equations of chiral $N=2 D=10$ supergravity, Nucl. Phys. B 226 (1983) 269 [inSPIRE].

[25] P.S. Howe and P.C. West, The complete $N=2, D=10$ supergravity, Nucl. Phys. B 238 (1984) 181 [inSPIRE].

[26] F. Apruzzi, J.C. Geipel, A. Legramandi, N.T. Macpherson and M. Zagermann, Minkowski $\times S^{2}$ solutions of IIB supergravity, Fortsch. Phys. 66 (2018) 1800006 [arXiv:1801.00800] [INSPIRE].

[27] E. D'Hoker, M. Gutperle and C.F. Uhlemann, Holographic duals for five-dimensional superconformal quantum field theories, Phys. Rev. Lett. 118 (2017) 101601 [arXiv: 1611.09411] [inSPIRE].

[28] M. Gutperle, A. Trivella and C.F. Uhlemann, Type IIB 7-branes in warped AdS $S_{6}$ : partition functions, brane webs and probe limit, JHEP 04 (2018) 135 [arXiv:1802.07274] [INSPIRE].

[29] O. Bergman, D. Rodríguez-Gómez and C.F. Uhlemann, Testing AdS $S_{6} / C F T_{5}$ in type IIB with stringy operators, JHEP 08 (2018) 127 [arXiv: 1806. 07898] [INSPIRE].

[30] M. Gutperle, C. Marasinou, A. Trivella and C.F. Uhlemann, Entanglement entropy vs. free energy in IIB supergravity duals for 5d SCFTs, JHEP 09 (2017) 125 [arXiv:1705.01561] [INSPIRE].

[31] M. Gutperle, C.F. Uhlemann and O. Varela, Massive spin 2 excitations in $A d S_{6} \times S^{2}$ warped spacetimes, JHEP 07 (2018) 091 [arXiv: 1805.11914] [INSPIRE]. 
[32] M. Fluder and C.F. Uhlemann, Precision test of $A d S_{6} / C F T_{5}$ in type IIB, arXiv: 1806.08374 [INSPIRE].

[33] S. Ferrara, A. Kehagias, H. Partouche and A. Zaffaroni, $A d S_{6}$ interpretation of $5 D$ superconformal field theories, Phys. Lett. B 431 (1998) 57 [hep-th/9804006] [INSPIRE].

[34] R. D'Auria, S. Ferrara and S. Vaula, Matter coupled $F(4)$ supergravity and the $A d S_{6} / C F T_{5}$ correspondence, JHEP 10 (2000) 013 [hep-th/0006107] [INSPIRE].

[35] P. Karndumri, Holographic RG flows in six dimensional $F(4)$ gauged supergravity, JHEP 01 (2013) 134 [Erratum ibid. 06 (2015) 165] [arXiv:1210.8064] [INSPIRE].

[36] P. Karndumri, Gravity duals of $5 D N=2 S Y M$ theory from $F(4)$ gauged supergravity, Phys. Rev. D 90 (2014) 086009 [arXiv:1403.1150] [INSPIRE].

[37] L.F. Alday, M. Fluder, P. Richmond and J. Sparks, Gravity dual of supersymmetric gauge theories on a squashed five-sphere, Phys. Rev. Lett. 113 (2014) 141601 [arXiv:1404.1925] [INSPIRE].

[38] L.F. Alday, M. Fluder, C.M. Gregory, P. Richmond and J. Sparks, Supersymmetric gauge theories on squashed five-spheres and their gravity duals, JHEP 09 (2014) 067 [arXiv: 1405.7194] [INSPIRE].

[39] L.F. Alday, P. Richmond and J. Sparks, The holographic supersymmetric Rényi entropy in five dimensions, JHEP 02 (2015) 102 [arXiv:1410.0899] [INSPIRE].

[40] N. Hama, T. Nishioka and T. Ugajin, Supersymmetric Rényi entropy in five dimensions, JHEP 12 (2014) 048 [arXiv:1410.2206] [INSPIRE].

[41] L.F. Alday, M. Fluder, C.M. Gregory, P. Richmond and J. Sparks, Supersymmetric solutions to Euclidean Romans supergravity, JHEP 02 (2016) 100 [arXiv: 1505. 04641] [INSPIRE].

[42] M. Gutperle, J. Kaidi and H. Raj, Janus solutions in six-dimensional gauged supergravity, JHEP 12 (2017) 018 [arXiv: 1709.09204] [INSPIRE].

[43] C.-M. Chang, M. Fluder, Y.-H. Lin and Y. Wang, Romans supergravity from five-dimensional holograms, JHEP 05 (2018) 039 [arXiv:1712.10313] [INSPIRE].

[44] M. Gutperle, J. Kaidi and H. Raj, Mass deformations of 5d SCFTs via holography, JHEP 02 (2018) 165 [arXiv: 1801.00730] [INSPIRE].

[45] M.J. Duff, B.E.W. Nilsson, C.N. Pope and N.P. Warner, On the consistency of the Kaluza-Klein ansatz, Phys. Lett. B 149 (1984) 90 [INSPIRE].

[46] P. Hoxha, R.R. Martinez-Acosta and C.N. Pope, Kaluza-Klein consistency, Killing vectors and Kähler spaces, Class. Quant. Grav. 17 (2000) 4207 [hep-th/0005172] [INSPIRE]. 